\title{
Pas de nouvelles, mauvaises nouvelles
}

\author{
Curtis F. Lavoie, MD
}

Voir les ARticles connexes AuX PAges 523 et 545

English Version on page 566

E

xercer la médecine d'urgence est un peu comme voler un avion à basse altitude ... dans les Rocheuses ... les yeux bandés. Vous pouvez essayer d'éviter le danger, mais les résultats de vos décisions sont inconnus. Jusqu'à ce que, bien sûr, l'avion s'écrase sur le mont Robson.

Par un jour calme de septembre, je me suis adonné à entendre un groupe d'infirmières et de médecins où je travaille qui parlaient d'un récent cas qui les avait fait « frémir ». Un garçonnet de 9 ans se présente à l'urgence, se plaignant d'un mal de tête. Il est vu par un médecin, traité avec du métoclopramide, puis renvoyé chez lui. Six heures plus tard, il revient à l'urgence et reçoit un diagnostic de méningite. Il doit être intubé et est admis à l'unité des soins intensifs. Tout le monde est sous le choc. Comment cela a-t-il pu se produire? Après une pause, un collègue se tourne vers moi et me dit : « Hé! Curtis, je pense que ton nom figurait sur son dossier. »

Ah! J'aurais donc dû écouter ma mère et devenir pilote à la place!

On a souvent fait un parallèle entre l'industrie aéronautique et la pratique de la médecine d'urgence ${ }^{1}$, mais je ne partage pas ce point de vue. D'une part, les pilotes, comme les médecins d'urgence, font des études très poussées et effectuent des stages rigoureux. Puis, ils travaillent sous beaucoup de pression, à toutes heures et dans des situations où le temps est limité. Ils doivent prendre des décisions ayant potentiellement des conséquences désastreuses. D'autre part, les pilotes n'ont qu'à regarder par la fenêtre pour obtenir de l'information de haute qualité sur les résultats de chacune de leurs décisions et, le résultat le plus important, un atterrissage réussi (ou pas) à une destination prévue, est facile à observer à la fin de chaque quart de travail (Fig. 1).
En médecine d'urgence, c'est tout le contraire. L'état de santé des patients évolue sur quelques jours ou semaines, ce qui signifie que des informations importantes relatives au diagnostic et au traitement peuvent n'apparaître qu'après que le patient quitte l'hôpital, est transféré à un autre service ou est admis à l'hôpital. En outre, habituellement, nous ne revoyons pas nos patients, et il n'existe aucun système pour nous dire si leur état s'améliore ou empire. À mon avis, en l'absence d'information sur les résultats de nos décisions, nous volons les yeux bandés.

Dans une certaine mesure, tous les médecins d'urgence doivent assumer que l'adage « pas de nouvelles, bonnes nouvelles $\gg^{2}$ s'applique, s'ils veulent avoir confiance en leurs décisions. Le problème avec cet adage, c'est qu'il suppose qu'il y a un impératif systémique pour nous laisser savoir quand les choses tournent mal. En d'autres termes : «Quelqu'un aurait déjà dit quelque

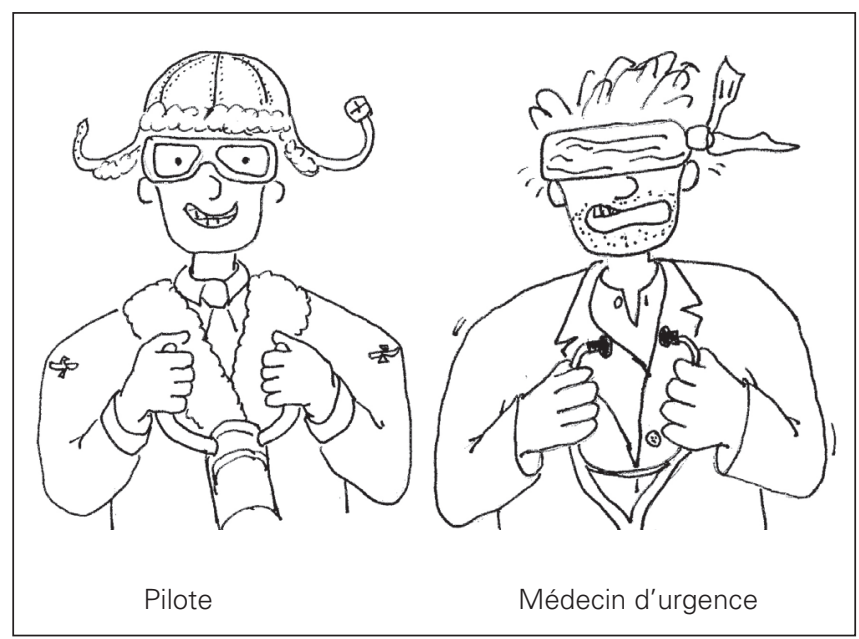

Fig. 1. L'aviation et la médecine d'urgence.

Service d'urgence, Hôpital Montfort, et Service des urgences, Centre hospitalier pour enfants de l'est de l'Ontario, Ottawa (Ontario) Les opinions exprimées dans les éditoriaux sont celles des auteurs; elles ne reflètent pas nécessairement celles du JCMU ou de l'Association canadienne des médecins d'urgence. 
chose. » Malheureusement, ce raisonnement est faussé Les patients ne peuvent pas souvent communiquer avec nous, des collègues pourraient hésiter à nous transmettre de mauvaises nouvelles et notre système de santé comporte peu de mécanismes de surveillance pour signaler, aux médecins, des résultats inattendus. L'argument «Je n'ai jamais été poursuivi en justice » est également faussé. Une poursuite en justice n'est intentée que si un patient souffre, qu'il y a une erreur médicale soupçonnée et une motivation à réparer le tort. La plupart des résultats n'atteignent pas ce seuil.

Selon Croskerry ${ }^{3}$, il est nécessaire de connaître l'issue des soins pour « étalonner » les instruments de prise de décisions des cliniciens, une idée provenant de la littérature des sciences de la décision². L'étalonnage permet aux praticiens d'améliorer la précision de leurs estimations de la probabilité après avoir obtenu des renseignements sur le devenir des patients. Si les résultats sont positifs, ils renforcent les comportements vis-à-vis des décisions; s'ils sont négatifs, ils forcent le médecin à réfléchir à sa décision et, éventuellement, à modifier ses comportements (Fig. 2). Le problème est que lorsque nous ne connaissons pas le devenir du patient, nous supposons qu'il est positif, ce qui renforce toutes nos stratégies diagnostiques et thérapeutiques, y compris celles qui ont échoué. Les erreurs sont renforcées au lieu d'être découragées, et les médecins continuent d'avoir recours à des stratégies inefficaces, voire dangereuses ${ }^{2,3}$. Une meilleure communication des données sur les résultats

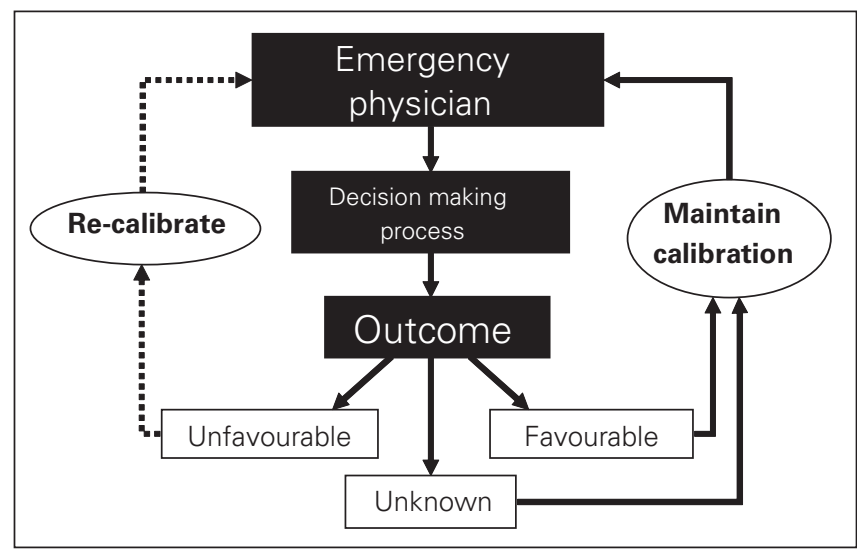

Fig. 2. L'information sur les résultats et l'étalonnage en médecine d'urgence : la plupart des résultats sont inconnus et cela peut renforcer toutes les stratégies de prise de décisions, même si elles sont erronées. Reproduit avec la permission. ${ }^{3}$

Veuillez noter: Nous n'avons pu obtenir à temps I'autorisation de reproduction de la version française de la figure. Veuillez donc consulter la version anglaise. Nous regrettons tout inconvénient que cela pourrait causer. pour les patients ferait ressortir les erreurs et offrirait la possibilité d'améliorer la précision du diagnostic, les choix thérapeutiques et la sécurité des patients.

En outre, l'étalonnage peut avoir d'autres utilités, notamment contribuer à améliorer l'efficacité clinique. En connaissant les résultats pour un pourcentage croissant de patients, les médecins changeraient progressivement leur approche pour n'utiliser que les indices cliniques et les tests les plus utiles pour atteindre les seuils diagnostiques ou thérapeutiques. Qui plus est, la satisfaction professionnelle peut être touchée : un manque d'information sur l'issue de la consultation peut miner le sentiment de valeur des médecins à l'égard de leur communauté (« Est-ce que je fais une différence dans la vie de mes patients? Qui sait? ») et avoir une incidence négative sur leur perception quant à l'auto-étalonnage ou leur expertise (« Suis-je bon dans ce que je fais? Qui sait?! »). L'absence d'information de qualité sur les résultats peut aussi nous mener indirectement à demander au patient de passer des tests inutiles pour apaiser nos besoins intellectuels ou rehausser notre confiance en nous. La transmission de plus d'information sur les résultats pourrait améliorer les deux à la fois.

Intuitivement, il est important de diriger son attention sur les résultats des décisions pour apprendre toute technique ou acquérir toute compétence. Les jardiniers ont besoin de voir leurs azalées fleurir, les chefs doivent goûter à leur bouillabaisse et les avocats ont besoin de lire leurs verdicts. Pourtant, même parmi les professionnels de la santé, les médecins d'urgence font bande à part. Les médecins de soins primaires suivent certains patients pendant des années, et les spécialistes admettent les patients à l'hôpital ou font un suivi auprès de leurs patients pour évaluer les résultats de leurs soins et traitements. Les pédiatres voient leurs patients grandir, les psychiatres voient leurs patients sourire, les urologues voient leurs patients faire pipi et les chirurgiens du côlon et du rectum ..., eh bien ..., je crois que vous comprenez. L'information sur les résultats est si fondamentale à la maîtrise de toute profession que, pour la plupart d'entre nous, cela va de soi. En médecine d'urgence, par contre, ce n'est pas le cas.

\section{CONCLUSION}

Après avoir écouté cette conversation sur le garçon atteint de la méningite, je suis allé tout droit à l'étage où il était hospitalisé. Il allait bien. En fait, il jouait à des jeux vidéo. J'ai regardé son dossier, pris quelques notes et suis retourné à mon travail. Ce qui est remarquable, c'est 
qu'en dépit de la gravité du diagnostic, ce n'est que par hasard que j'ai obtenu de l'information sur ce patient. Le problème, c'est que c'est comme cela que ça se passe.

L'information sur les résultats des soins ne devrait pas être laissée au hasard : les médecins d'urgence ne peuvent pas supposer que si l'état de santé d'un de leurs patients se détériore, qu'ils vont en entendre parler. De plus, « en entendre parler » semble essentiel pour étalonner les outils de prise de décisions cliniques, éviter les erreurs et améliorer de façon générale la pratique médicale. C'est grâce aux données sur les résultats de décisions que la plupart des autres professionnels deviennent des experts. Alors, au nom de l'expertise et de la sécurité des patients, nous devons comprendre l'importance de ces données et améliorer leur communication aux médecins d'urgence, surtout lorsque les choses tournent mal. C'est seulement après cela que nous pourrons nous débander les yeux et avoir un vol agréable.
Conflits d'intérêts : aucun déclaré.

Mots clés : information sur les résultats du traitement, qualité des soins de santé, médecine d'urgence, techniques de soutien des décisions, information sur les résultats

\section{RÉFÉRENCES}

1. Kohn LT, Corrigan JM, Donaldson MS, editors. To err is buman: building a safer bealth care system. Washington (DC): National Academy Press; 2000.

2. Rudolph JW, Morrison JB. Sidestepping roadblocks: a feedback model of diagnostic problem solving. Am 7 Med 2008; 121:S34-7.

3. Croskerry P. The feedback sanction. Acad Emerg Med 2000; 7:1232-8

Correspondance : $\mathrm{D}^{\mathrm{r}}$ Curtis Lavoie, Hôpital Montfort, 713, chemin Montréal, Ottawa ON K1K 3E9; curtislavoie@gmail.ca

\section{Errata}

In an article entitled, "Opposites attract: a case of magnet ingestion" published in the September issue (CJEM 2009;11:493-5), there was an error in the following sentence in the Discussion section:

In reviewing the previous case reports and all required surgical intervention, ${ }^{47}$ it is not clear how often cases of multiple magnet ingestions require no intervention. We were unable to find any cases of these reported in the literature. However, the number of case reports documenting severe complications from single battery ingestion suggests that morbidity is not uncommon.

The sentence should read as follows:

In reviewing the previous case reports and all required surgical intervention, ${ }^{47}$ it is not clear how often cases of multiple magnet ingestions require no intervention. We were unable to find any cases of these reported in the literature. However, the number of case reports documenting severe complications from single magnet ingestion suggests that morbidity is not uncommon.

In the September issue of CJEM, the education and affiliation for John Fahimi (Stenstrom R, Grafstein E, Romney M, Fahimi J, Harris D, Hunte G, Innes G, Christenson J. Prevalence of and risk factors for methicillin-resistant Staphylococcus aureus skin and soft tissue infection in a Canadian emergency department. CJEM 2009;11:430-8) were inadvertently listed incorrectly. The name and affiliation should read, "Jahan Fahimi, MD, MPH. Dr. Fahimi is affiliated with the Alameda County Medical Center, Highland Hospital, Oakland, Calif."

One of the authors in an article published in our September issue (Schull MJ, Vaillancour S, Donovan L, et al. Underuse of prehospital strategies to reduce time to reperfusion for ST-elevation myocardial infarction patients in 5 Canadian provinces. CJEM 2009;11:473-80.) was inadvertently listed as Lucy J. Boothroyd, PhD. In fact, this author should have been listed as Lucy J. Boothroyd, $\mathrm{PhD}$ candidate. 